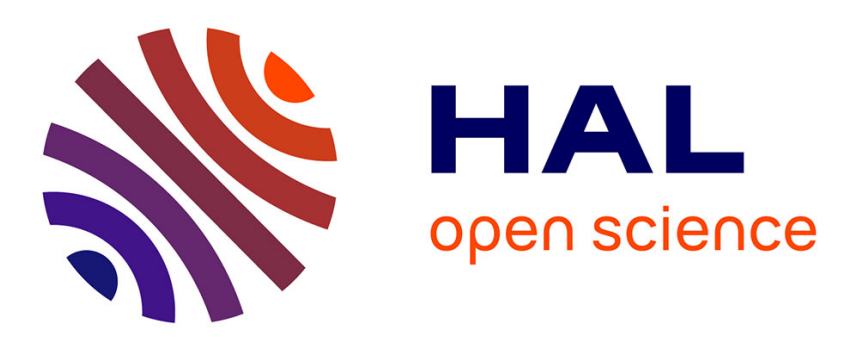

\title{
Album papier, album numérisé : une dialectique outil-objet avec le TBI
}

\author{
Isabelle Bastide, Cédric Fluckiger
}

\section{To cite this version:}

Isabelle Bastide, Cédric Fluckiger. Album papier, album numérisé: une dialectique outil-objet avec le TBI. Villemonteix F., Baron G.-L., Béziat J. L'école primaire et les technologies informatisées. Des enseignants face aux TICE, Presses Universitaires du Septentrion, pp.47-58, 2016, 978-2-7574-1277-0.

hal-01375362

\section{HAL Id: hal-01375362 \\ https://hal.univ-lille.fr/hal-01375362}

Submitted on 3 Oct 2016

HAL is a multi-disciplinary open access archive for the deposit and dissemination of scientific research documents, whether they are published or not. The documents may come from teaching and research institutions in France or abroad, or from public or private research centers.
L'archive ouverte pluridisciplinaire HAL, est destinée au dépôt et à la diffusion de documents scientifiques de niveau recherche, publiés ou non, émanant des établissements d'enseignement et de recherche français ou étrangers, des laboratoires publics ou privés. 
Album papier, album numérisé : une dialectique outil-objet avec le TBI

Référence : Bastide Isabelle, Fluckiger Cédric, (2016), Album papier, album numérisé: une dialectique objet-outil avec le TBI, in Villemonteix F., Baron G.-L., Béziat J. (dirs), L'école primaire et les technologies informatisées. Des enseignants face aux TICE, Villeneuve D'Ascq, Presses Universitaires du Septentrion, p. 47-58.

\section{Bastide Isabelle}

Doctorante, Circeft- Escol, Université Paris 8, France

Isabellelaure.bastide@gmail.com

\section{Fluckiger Cédric}

Maître de conférences, Théodile-CIREL (EA4354), Université Lille 3, France

Cedric.fluckiger@univ-lille3.fr

\section{Introduction}

Cette communication vise à présenter les premiers résultats d'une recherche exploratoire sur les usages du tableau blanc interactif (TBI) en primaire. Nous cherchons à étudier, d'un point de vue didactique, l'utilisation de TBI lors de lectures partagées d'albums de littérature de jeunesse en classe de CP.

Cette communication s'inscrit dans le cadre des premiers travaux d'une recherche menée par l'équipe Théodile-CIREL (Lille 3), commanditée par l'Inspection de Lille et financée sur réserve parlementaire du Sénat, visant à identifier, décrire et caractériser les ressources didactiques et pédagogiques utilisées par les enseignants, en particulier lorsqu'ils sont confrontés à l'usage d'un TBI. La question que nous posons plus spécifiquement dans cette communication est celle des modifications et adaptations que peuvent subir les ressources au sein de la classe durant différentes séances, en fonction des possibilités interactives offertes par le TBI. Nous prenons le cas du travail d'une enseignante en classe CP, qui utilise, en lieu et place d'une méthode de lecture traditionnelle, des albums dans leur version papier ainsi que leur version numérisée. Nous confrontons l'analyse des séances observées d'une part à celle de l'entretien effectué avec l'enseignante et d'autre part à l'analyse du contenu des albums dans leurs deux versions.

\section{TBI, ressources didactiques et lectures d'album : cadres théoriques}

Les recherches sur les technologies numériques éducatives posent de manière fréquente la question de l'instrumentation des apprentissages, des genèses instrumentales (Rabardel, 1995) à l'œuvre ; les discours, 
activités instrumentées peuvent être étudiés dans différentes perspectives. Le TBI a par exemple été interrogé dans sa relation au travail enseignant (Numa Bocage et al., 2011). En revanche, une perspective didactique visant à mettre en relation les usages d'outils numériques pour l'élève ou l'enseignant avec les contenus d'enseignement et d'apprentissages est plus rarement mise en œuvre (Fluckiger, 2012). Il nous semble pourtant que la diffusion de certaines technologies modifie singulièrement l'organisation des contenus, leur appréhension, perception, appropriation par les différents acteurs. C'est ce point de vue qui sera adopté ici, le TBI étant moins considéré dans sa dimension instrumentale que dans ses potentialités de structuration et/ou de reconfiguration partielle de la situation didactique.

\section{La question des ressources des enseignants}

Sans tomber dans un déterminisme technologique, ou dans l'illusion de nouveauté qui accompagne le développement de toute technologie éducative (voir Bruillard, 1997 ; Baron et al., 2009 ; Baron, 2013), nous formulerons l'hypothèse que le TBI peut avoir des effets didactiques, notamment quant à l'usage et au devenir des ressources et documents didactiques et pédagogiques utilisés par les enseignants. Ces effets sont à relier d'une part à la multiplicité des sources possibles pour les ressources et les documents pédagogiques, d'autre part à l'évolution des conceptions sur les contenus et pratiques pédagogiques qui accompagnent l'appropriation de nouvelles technologies éducatives (Mishra et Koehler, 2006). Le TBI donne ainsi la possibilité aux enseignants de multiplier les formats des documents proposés aux élèves : images et graphiques, textes, exercices, vidéos, sons, posant de manière accrue la question des ressources, de leur origine et de leurs transformations éventuelles.

Nous entendons ici la notion de ressources éducatives comme tout ce qu'un enseignant consulte dans l'objectif de préparer un cours (manuel, site, fiche de préparation, document sonore, vidéo ou photo, article de journal, fiches élève ou fiche pour l'enseignant, directement pour les élèves ou pour eux, documents authentiques ou construits, sans distinguer a priori ressource numérique ou autre (voir Bibeau, 2005), et en distinguant, à la suite de Gueudet et Trouche (2010), ressource et document (le dernier ayant fait l'objet d'un travail de genèse documentaire).

Ce qui nous intéresse plus précisément, c'est le devenir des documents utilisés en classe, dans le cas de lectures partagées (Frier, 2006 ; Grossmann, 1996) d'albums numérisés en classe de CP.

\section{Les lectures partagées en classe de CP}

Les lectures partagées mettent en situation de coprésence différents acteurs : celui qui lit et ceux à qui on lit. Une des particularités de ces lectures réside dans leur mode de passation (Grossmann 1996 ; Frier, 2006 ; Bastide, Joigneaux, 2014). Il existe non pas un type de lecture partagée, mais une pluralité, chacun 
d'eux possédant des spécificités faisant plus ou moins intervenir, dans un cadre scolaire et/ou familial, le lecteur, les élèves, le support (l'album) et les contenus de ce dernier. Dans le questionnement qui est le nôtre, le point crucial est le fait que certaines de ces lectures accordent une place centrale à l'objet livre, celui-ci constituant le point clef d'une entrée dans l'écrit (voir par exemple les lectures enquêtes ou les lectures dialogiques dans Bastide et Joigneaux, 2014) en mettant en avant les gestes de lecture nécessaires à la compréhension des contenus dans leur globalité (aller-retour entre texte et images, les retours au texte, le rôle et les effets du changement de page dans l'avancée du récit...).

Or les recherches précédentes se sont basées principalement sur l'utilisation d'un support papier. Se pose en conséquence la question de l'utilisation d'un support numérique pour ces lectures ? L'objet livre n'est alors plus nécessairement présent dans sa forme matérielle lors de tous les exercices ; texte et images sont toujours présents mais ont changés de format et, étant dans une certaine mesure mis à distance des élèves, le rapport à l'objet-livre change. Dans quelle mesure, comment ?

\section{Une problématique ancrée en didactique}

Pour répondre à ces questions, nous formulons l'hypothèse que dans les albums numérisés, l'agencement des contenus a été modifié, lors d'un travail de «genèse documentaire » de l'enseignant (Gueudet et Trouche, 2008), au profit par exemple d'insertions d'animations, de parenthèses narratives (lien vers un ajout narratif)... Cet agencement particulier crée des conditions différentes lors des situations de lectures partagées, situations en partie modifiées, transformées par le support (album numérique/ album numérisé) et ses contenus mais également par le TBI qui est à la fois l'outil (au sens didactique du terme) qui permet ces lectures ainsi qu'un élément qui les conditionne. Il jouerait ainsi un rôle actif dans la constitution du milieu didactique de ces séances de lectures partagées.

Une phase d'observation exploratoire d'utilisation du TBI dans différentes matières scolaires en primaire nous a permis de poser la question de la place tenue par le TBI dans la constitution de différents milieux didactiques ainsi que dans l'institution des statuts des différents acteurs (élèves, maître). Nous avons ensuite observé une enseignante de classe de CP dans la région lilloise. Nous fondons notre analyse du travail de cette enseignante sur un corpus exploratoire, composé de trois segments :

- l'observation de huit séances consacrées à la lecture d'albums ;

- $\quad$ un entretien semi directif enregistré avec l'enseignante ;

- les albums étudiés en classe et l'ensemble du matériel pédagogique de l'enseignante, surtout et y compris ce que nous appelons la version numérisée de l'album.

Ainsi, c'est bien la double confrontation des pratiques de classe observées instrumentées, avec le TBI ou le support papier d'une part, et avec les contenus présents dans l'album d'autre part (contenus d'entrée 
dans la langue écrite, compétences lecturales, contenus sur le monde, connaissances possibles d'ordre scientifique ou non (Bastide, 2013), mais aussi valeurs, rapports à (Reuter, 2013), etc.) qui marque l'ancrage théorique didactique de notre question.

\section{Plan de l'article}

Notre analyse des évolutions subies par les documents didactiques de l'enseignante s'articule en 4 axes. Dans un premier temps, nous montrerons que le travail d'adaptation effectué par l'enseignante consiste en réalité en la conception et la réalisation d'une version " autre » de l'album. Nous cherchons ensuite à spécifier les caractéristiques de la version numérisée de l'album, notamment en ce qu'elle permet à l'enseignante de mener un double travail dans et sur le contenu. Il s'agit, dans la version numérisée, d'une appréhension particulière du contenu, rendue possible par les possibilités interactives du TBI. Troisièmement, nous montrons que la version numérisée permet de faire le lien entre les différentes activités didactiques de lecture et écriture. Enfin, nous montrerons comment le TBI et la version numérisée de l'album qui y est présenté s'insère dans le contexte didactique plus large de la classe, la version numérisée entrant en résonnance avec le vécu scolaire des élèves.

\section{Résultats}

\section{De l'adaptation à la création}

Le travail d'adaptation qu'effectue l'enseignante consiste en la conception et la réalisation d'une version «autre » de l'album, adaptée au TBI d'une part, aux élèves et à leurs besoins d'autre part, ainsi qu'aux objectifs pédagogiques de l'enseignante. Les contenus de l'album numérisé, sont ainsi remaniés, réorganisé pour être (re)mis en scène en donner naissance à une version numérisée de ce dernier, version qui a fait l'objet d'une « genèse documentaire » (Gueudet et Trouche, 2008).
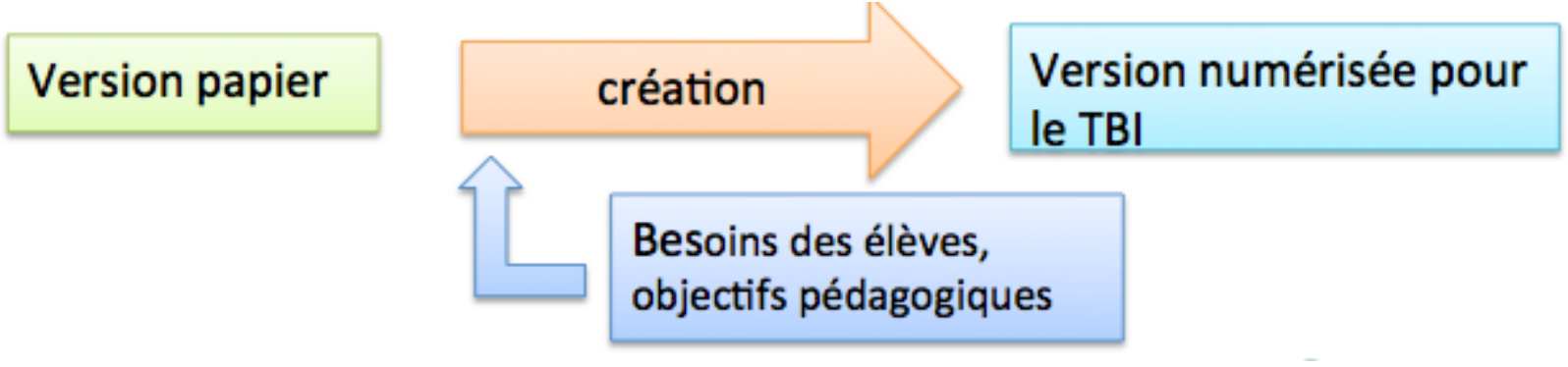

Le support présenté sur le TBI a une existence, un sens dans la situation dans laquelle il est présenté et seulement dans celle-là dans la mesure où le texte n'est pas nécessairement présent là où le support papier l'attend... L'absence de texte perturberait considérablement la lecture de l'album dans un autre 
contexte que celui de cette classe, ce qui n'est pas le cas ici dans la mesure où cette absence devient signifiante pour les élèves : elle les met de fait en situation d'écriture.

Par exemple, lors d'une séance de lecture, l'enseignante présente l'image de la double page suivante :

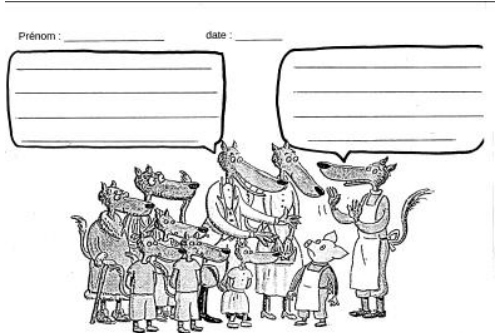

Le texte a été effacé par ses soins. "Voici l'image suivante, que peuvent bien dire les personnages, imaginez ce que peuvent dire les loups ». Elle ajoute : «Ça c'est la famille loup, essayez d'imaginer ce qu'ils sont en train de se dire ».

Les enfants écrivent leur propre texte de manière individuelle puis lors de la mise en commun, 1 'enseignante écrit le texte des enfants à la place du texte original. La création du support vierge de texte se prolonge donc, par la création collective par les élèves d'un texte possible, qui fait sens dans l'histoire. L'enseignante conclut cette activité en disant «je vous montre la suite, on va voir si ça va ». Elle ne montre pas cette double page, elle reprend la lecture magistrale à la double page suivante. Les élèves n'auront la lecture du texte original qu'à la fin de la séance, sous forme de bilan.

Ce nouveau support nécessite donc un dévoilement progressif ainsi que le remplissage des «blancs » qu'il présente, blancs placés en général à des moments clefs de l'action.

Cette version numérisée présente dès lors un caractère éphémère au double sens où elle n'existe que dans un contexte précis, pour cette classe, mais aussi au sens où cette version « autre » sera modifiée au cours du travail effectué (voir infra). En effet, entre le moment de sa présentation initiale (un ensemble de pages avec des trous, des ajouts, des manques, des caches) et la fin de son utilisation, la version numérisée a considérablement évolué. Des blancs ont été comblés, des animations se sont ajoutées au gré des besoins d'explications lexicales et/ou culturelles des élèves... 


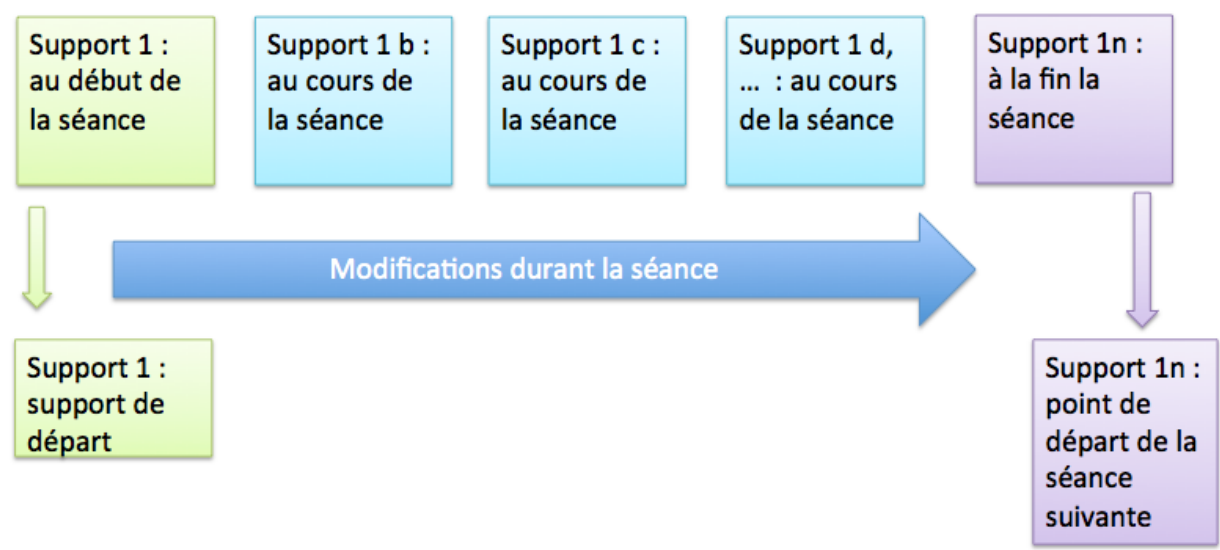

Par exemple, lors d'une séance précédente l'enseignante s'étant rendue compte que le mot valse était inconnu des élèves, elle commence la séance en montrant un extrait de vidéo de valse. Ce qu'elle n'avait pu faire en raison de l'absence de connexion internet. Le lien de la vidéo reste dans le support de cours créé à partir de l'album et ce voit ainsi enrichi.

A l'inverse de sa jumelle, la version papier, qui présente un caractère immuable et fixe, cette version numérisée évolue donc, change mais ne revient pas sur ses pas. Les modifications apportées restent. Le document, dans son contenu, est donc éphémère à plus d'un titre : il n'existe que dans un contexte précis, se métamorphose au fil des séances,... En résumé, on se trouve confronté à un support initial créé en amont de la séance qui sera ensuite modifié au cours de celle-ci.

\section{Agir dans agir sur}

Dans un deuxième temps, nous cherchons à spécifier les caractéristiques de la version numérisée de l'album. Elle permet notamment à l'enseignante de mener un double travail dans et sur le contenu. Il s'agit donc, dans la version numérisée, d'une appréhension particulière du contenu, rendue possible par les possibilités interactives du TBI.

Parfois le contenu de l'album est présenté tel quel, sans modification, sans ajout ce constitue un cas plutôt rare. Le plus souvent, comme nous l'avons décrit plus haut, le contenu de l'album a fait l'objet d'une adaptation; adaptation, nous l'avons dit, se poursuit en cours d'utilisation. Ce qui nous intéresse ici c'est ce qui est modifié, et pourquoi.

Parfois l'enseignante agit sur le contenu : le texte de la page de l'album qu'elle présente est masqué par un cache, qu'elle dévoile progressivement mais elle peut également confronter les élèves à un cache mobile se déplaçant sur le texte ou une image sur l'image, comme le faisceau d'une lampe. 


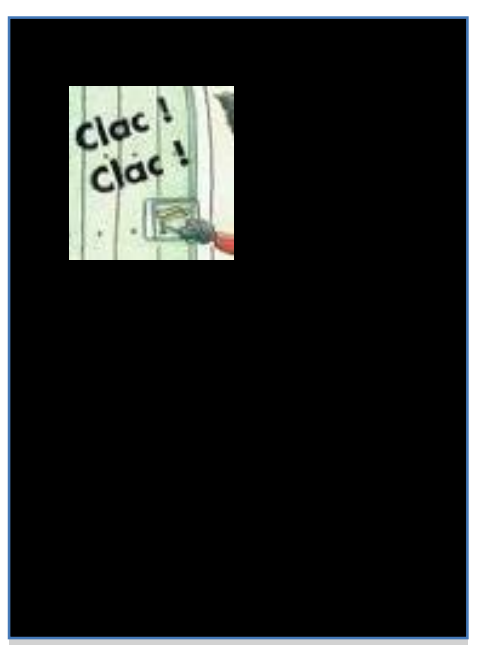

Les actions décrites ici sont des actions que l'on peut qualifier d'actions qui ont lieu en surface, le contenu est modifié à sa surface, autrement dit c'est sa présentation générale, son aspect extérieur qui se différencie de la version papier. On parle donc bien d'actions sur.

Un autre cas de figure se présente lorsque l'enseignante agit non plus sur mais dans le texte. On assiste alors à la création d'un texte comme dans l'exemple cité précédemment « imaginer ce que se disent les personnages », ou lorsqu'elle cache le texte, et demande aux élèves d'écrire un dialogue alors qu'à l'origine il s'agissait d'un texte narratif.

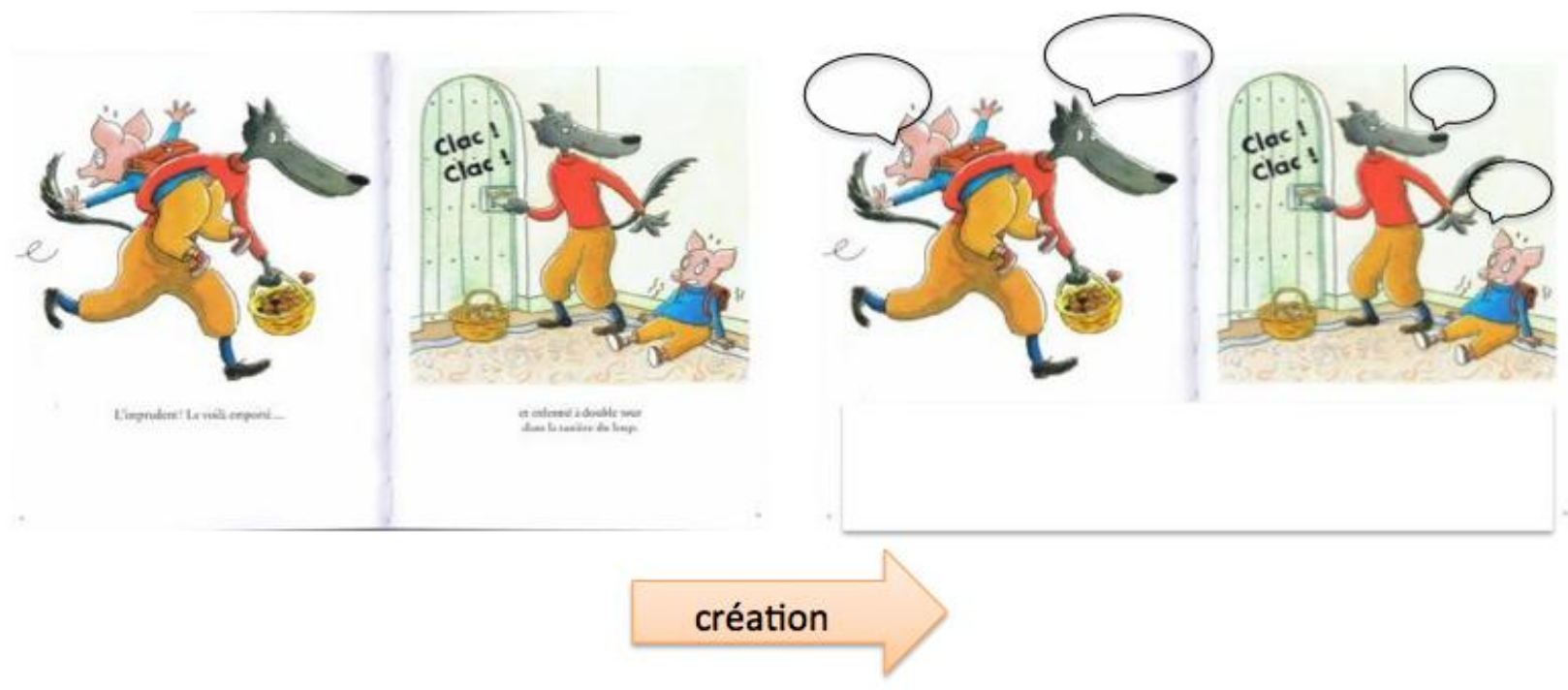

D'autres situations amènent ce type d'actions dans, par exemple la modification du texte d'origine qui se fait en direct sous le regard des élèves selon leurs besoins ou ceux de la situation: mots mis en couleur, surlignés, barrés, mots fragmentés pour faciliter la lecture. L'enseignante agit alors à l'intérieur même du support, ici du texte, le contenu de l'album se 
trouve alors modifié dans son contenu même. On est bien ici sur des actions internes des actions dans.

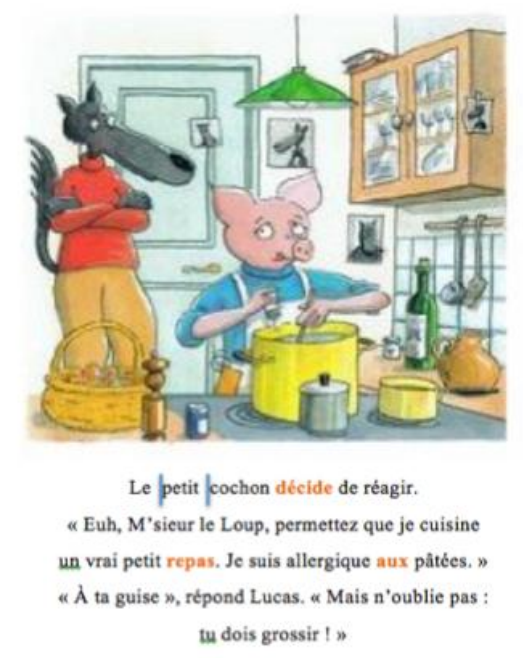

Ce qui est vrai pour le texte l'est pour les images qui peuvent elles aussi faire l'objet d'actions sur et/ou dans.

\section{Passage des activités}

La version numérisée, si elle n'est pas autonome, permet de faire le lien entre les différentes activités didactiques de lecture et d'écriture. Le passage d'une activité à l'autre devient une « nécessité » à l'avancement de la séance : la production d'un écrit conditionne la présentation de la suite du texte. Le même support, l'album numérisé, sert ainsi différents objectifs pédagogiques au sien d'une même séance.

\section{D'une activité à l'autre}

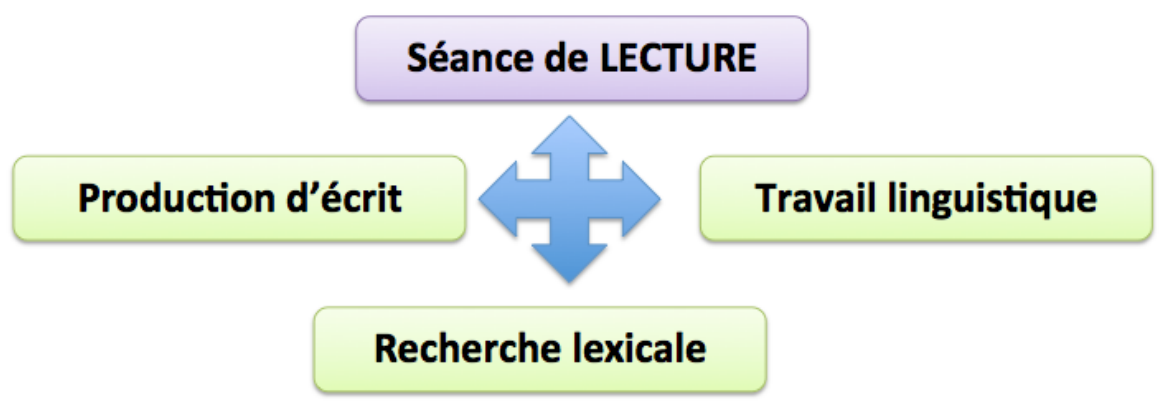

Avec la création d'un nouveau support de lecture, le passage d'une activité de lecture à une activité par exemple de production d'écrit devient une nécessité, afin d'avancer dans la lecture du 
texte. Lorsqu'une page est présentée alors que le texte est caché voire effacé, l'activité de production d'écrit devient une nécessité dans la mesure où l'image ne se suffit pas à elle-même.

En effet, ces activités sont généralement présentées aux élèves dans le cas où le texte et l'image ont une relation complémentaire (chacun des deux apporte une information différente, Isabelle Bastide, 2013 ; à partir des catégories de Grossmann, 1996).

Avec le TBI, les activités s'enchainent ainsi de manière fluide, selon une logique de narrativité. Le fait que ces activités (lecture, écriture, dessin) se déroulent sur un même support physique, le TBI, rend le passage de l'une à l'autre rapide, et probablement inconscient pour les élèves. Il ne s'agit pas de dire aux élèves : «posez le livre, prenez une feuille, vous allez écrire un texte », l'écriture est inscrite dans l'activité même de lecture.

\section{Le support dans la classe}

Enfin, nous nous sommes intéressés à la manière dont le TBI et la version numérisée de l'album qui y est présenté s'insèrent dans le contexte didactique plus large de la classe, la version numérisée entrant alors en résonnance avec le vécu scolaire des élèves.

Le TBI, ou plus exactement le support créé dans la séance trouve sa place parmi les autres « objets » présentes dans la classe, et auxquels les élèves peuvent recourir durant les séances.

\section{Le support dans la classe}
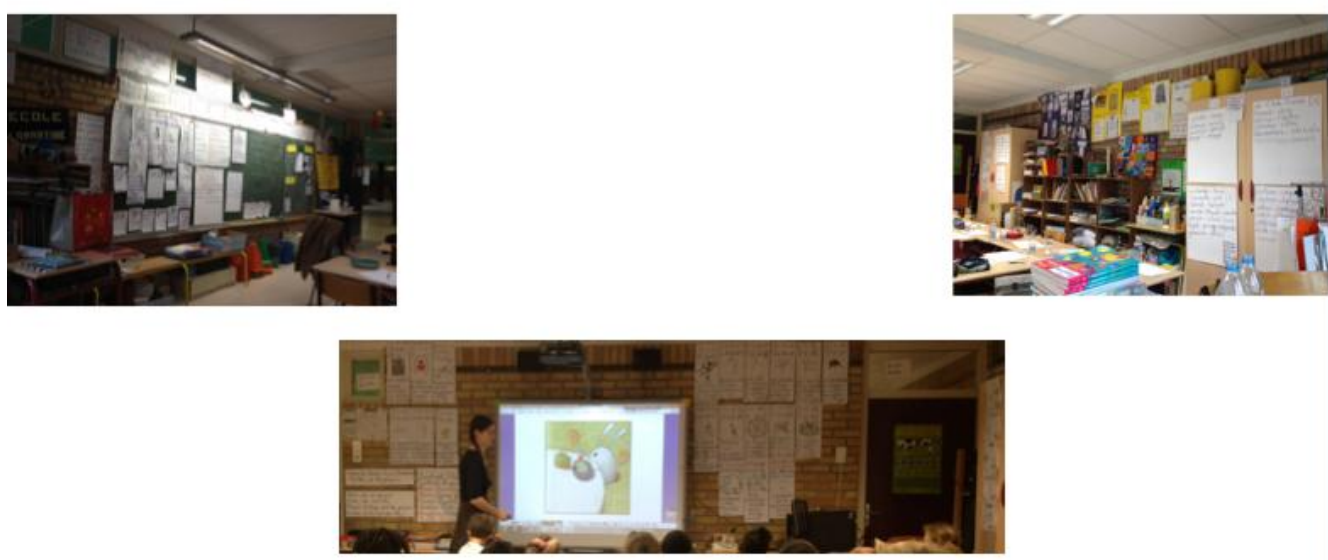

Bien que l'élément principal de la séance soit bien la version numérisée, le but de la séquence, exprimé par l'enseignante durant l'entretien, est bien l'utilisation de la version papier de l'album ; « le but c'est l'album ». 
En effet, si la version papier est une version autonome de l'album, le support créé pour le TBI ne l'est pas. Son utilisation vient ainsi en complément de l'objet livre, et s'en nourrit pour en faire découvrir le contenu aux élèves.

Nous supposions que le rapport à l'objet livre pouvait être modifié chez les élèves dans la mesure où l'objet livre n'étant alors plus présent dans sa forme matérielle, le texte et l'image se trouvaient mis à distance des élèves puisque qu'ils avaient changé de format.

En réalité, on ne lit pas au TBI pour lire, le TBI ramène l'objet livre au centre de l'activité en tant qu'outil et objectif à atteindre. Le livre reste le support mémoriel source d'informations, d'une gestuelle plus facile à conscientiser que sa version numérisée sur TBI pour laquelle les gestes restent virtuels.

Au cours des séances de lecture que nous avons observées, le livre est mobilisé tant comme objet que comme outil :

- la dimension de l'album comme objet est particulière présente lors de la première séance qui vise la découverte du livre. En effet, l'enseignante nomme les différentes composantes du support : auteur, première de couverture...;

- la fonction outil de l'album est quant à elle mobilisée lorsque l'objet d'apprentissage est présenté sur le TBI. Le livre devient alors l'outil de référence, de validation, d'aide... L'enseignante enjoint par exemple les élèves à se référer au livre papier pour vérifier l'écriture d'un mot.

L'utilisation de la version numérisée peut également être envisagée comme un outil permettant la découverte et l'appropriation progressive de l'album dans sa version papier par les élèves. En effet, si le livre dans sa globalité peut effrayer certains élèves dans la mesure où beaucoup d'informations se trouvent instantanément portées à la connaissance des élèves, la version numérisée présente le contenu étape par étape, selon une progression élaborée qui les acculture peu à peu à l'intégralité du contenu de l'album. Album qu'ils apprécieront ensuite de manière autonome dans sa version papier.

Le TBI prend aussi sa place dans l'ensemble des supports disponibles au sein de la classe. Aux cotés du TBI, il y a les affichages, un tableau noir. Lorsque l'enseignante veut qu'un écrit 
reste au delà de la page affichée, elle a recours au tableau noir. Lorsqu'un élève se trompe sur l'orthographe d'un mot, elle renvoie entre autre aux affiches.

Si nous nous détachons des supports pour aller vers les contenus mis en jeu dans les séances que nous avons observées, nous avons pu identifier différents types de contenus. Ces derniers font appel à différents supports, et s'adressent parfois au collectif, parfois à un enfant particulier... Nous sommes finalement sur des alternances collectif/individuel, supports virtuels (projections au TBI)/ supports matériels (tableaux noir, affiches...).

Nous avons ainsi identifié cinq types de contenus différents :

- Les contenus culturels renvoient au domaine culture littéraire et sont uniquement pris en charge par l'objet livre. Ils s'y réfèrent directement ;

- les contenus linguistiques qui consistent en un travail sur la langue sont pris en charge par le TBI uniquement, le livre n'intervient pas ou extrêmement peu. Ce type de contenus est principalement destiné au groupe classe dans son intégralité ;

- les contenus lecturaux consistent en un travail autour du code et du sens et sont d'une part pris en charge par le TBI et l'affichage pour ce qui relève du code et d'autre part par le TBI et l'album pour ce qui concerne le sens. Le travail sur le code est collectif tandis que es activités qui portent sur le sens peuvent aussi bien être individuelles que collectives ;

- les contenus d'ordre procédural (tourner les pages, se référer au livre...) qui relèvent avant tout d'un savoir-faire sont élaborés à partir de l'album papier et sont individuels ;

- les contenus de valeur et moraux s'adressent au groupe classe dans sa totalité peuvent être pris en charge par le TBI ou l'album papier mais aussi par d'autres albums lus précédemment qui se trouvent mis en réseau.

Le tableau suivant présente un récapitulatifs des différents types de contenus et de leurs modalités de prise en charge.

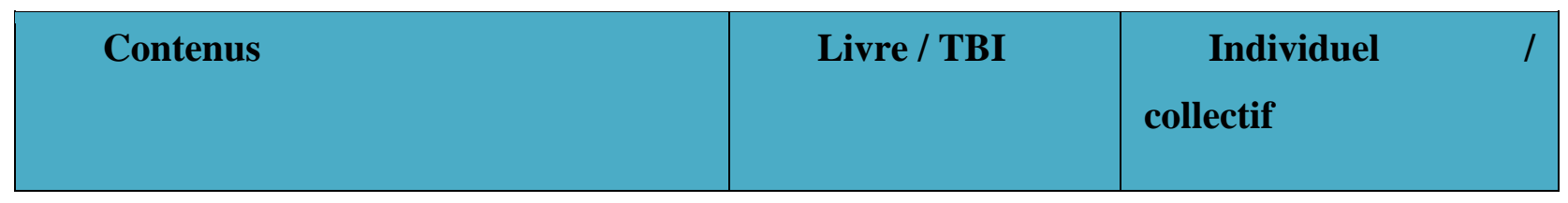




\begin{tabular}{|l|c|c|}
\hline $\begin{array}{l}\text { Contenus relatifs à une culture } \\
\text { littéraire }\end{array}$ & Album papier & collectif \\
\hline Contenus linguistiques & TBI & collectif \\
\hline Contenus lecturaux: le code & TBI & collectif \\
\hline Contenus lecturaux: le sens & Album papier et & semi-collectif \\
\hline Contenus procéduraux & TBI & \\
\hline Contenus de valeurs et moraux & Album papier & individuel \\
\hline & TBI & collectif \\
\hline
\end{tabular}

Nous avons fait le pari, pour échapper aux écueils mentionnés plus tôt, d'un ancrage didactique, c'est à dire d'une attention aux contenus.

En caractérisant les adaptations subies par le support d'origine vers le support présenté et travaillé en classe, nous avons vu que ces adaptations relevaient d'un double travail du contenu : action sur le texte, et action dans le texte. Nous avons montré que ce travail se prolonge dans la séance...

Enfin, nous avons montré que le support numérisé n'a pas d'existence autonome : l'objet livre reste bien central dans ce qui fait le contenu même des séances : la construction d'un rapport à la lecture, qui continue de passer par l'objet livre.

\section{Conclusion}

Notre entrée didactique, attentive aux contenus d'enseignement/apprentissages, nous a conduit à examiner la place des supports et ressources utilisées en classe, lors de séances de lectures partages 
d'albums en classe de CP. Cette prise en compte des contenus fait ainsi apparaître les jeux dialectiques entre outils et objets de l'apprentissage, déjà identifiés en didactique des mathématiques par Douady (ref).

Sans entrer dans le discussion de l'efficacité des dispositifs et des outils (Chaptal, 2001), il nous semble que le TBI modifie la place, le statut et le devenir des ressources didactiques employées en classe. En effet, pour être adaptées au TBI, les ressources documentaires, ici un album de littérature de jeunesse, font l'objet d'un processus de genèse documentaire (Gueudet et Trouche, 2010) qui se poursuit au cours même de la séance. Les ressources didactiques pour l'enseignement ne peuvent dès lors plus être pensées comme des «sources » préexistant a priori à la situation de classe, mais précisément comme des resources, sans cesse réélaborées, réadaptées par un double travail de surface et dans le contenu même de l'album.

\section{Bibliographie}

Baron G.-L. (2013). Enseignants, technologie éducative et techno-réformateurs. Vers une société sans écoles? Recherches en Didactiques, $n^{\circ} 16$, p. 59-73.

Baron G. L, Bruillard E. et Pochon L. O. (2009). Enjeux didactiques de l'informatique et de ses outils : vingt ans après : regards sur un cheminement, Informatique et progiciels en éducation et en formation : continuités et perspectives, G.-L. Baron, E. Bruillard \& L.-O. Pochon (dir.), Lyon, INRP, p.917.

Bastide I. (2013). Les connaissances possibles dans les albums de littérature de jeunesse : la transposition sémiotique des savoirs de référence au savoir imagé. Mémoire de M2, DEFF, Université Lille 3.

Bastide I. et Joigneaux C. (2014). Littératie précoce, albums et lectures partagées à l'école maternelle, Spirale, n॰53, p. 9-19.

Bibeau R. (2005).Les TIC à l'école: proposition de taxonomie et analyse des obstacles à leur intégration, Revue électronique de l'EPI, consulté le 15 novembre 2013 : http://www.epi.asso.fr/revue/articles/a0511a.htm.

Bruillard E. (1997). Les machines à enseigner. Paris, Hermès.

Chaptal A. (2001). L'investissement en vaut-il la peine ?, Revue électronique de l'EPI, consulté le 15 décembre 2013 : http://www.epi.asso.fr/revue/100/ba0p053.htm. 
Chevallard Yves (1985/1991). La Transposition didactique: du savoir savant au savoir enseigné. Grenoble, La pensée sauvage.

Douady R. (1986). Jeux de cadres et dialectique outil-objet, Recherches en Didactiques des Mathématiques, Vol 7/2.

Fluckiger C. (2012). Colloque JOCAIR 2012 : apprentissages instrumentés en réseaux, quel sujet apprenant ?, Distances et médiations des savoirs, $\mathrm{n}^{\circ} 1$ | 2012, consulté le 15 décembre 2013 : http://dms.revues.org/150.

Frier C. (dir.) (2006). Passeurs de lecture : lire ensemble à la maison et à l'école, Paris, Rets.

Grossman F. (1996), Enfances de la lecture - manières de faire, manières de lire à l'école maternelle, Bern, Peter Lang.

Gueudet G. et Trouche L. (2008) « Du travail documentaire des enseignants : genèses, collectifs, communautés », Éducation et didactique [En ligne], vol 2 - n³ | Décembre 2008, mis en ligne le 01 décembre 2010, consulté le 15 novembre 2013 : http://educationdidactique.revues.org/342

Gueudet G. et Trouche L. (2010). Ressources en ligne et travail collectif enseignant : accompagner les évolutions de pratiques, Actes du congrès de l'AREF, Université de Genève.

Mishra, P. et Koehler, M. J. (2006). Technological pedagogical content knowledge: A framework for integrating technology in teacher knowledge. Teachers College Record, 108(6), 1017-1054. Consulté le 15 décembre 2013 : http://mkoehler.educ.msu.edu.

Numa Bocage L., Clauzard P. et Monchaux P. (2011). Utilisation du TBI et transformations du travail enseignant, Colloque INRP "Le travail enseignant au XXIe siècle", Lyon, 16-18 mars 2011.

Rabardel P. (1995). Les hommes et les technologies: approche cognitive des instruments contemporains, Paris, Armand Colin.

Reuter Y. (dir) (2013). Dictionnaire des concepts fondamentaux des didactiques, Bruxelles, De Boeck. 